\title{
A study of management of distal femoral fractures with intramedullary supracondylar nail
}

\author{
Shriniwas Yemul ${ }^{1}$, Milind M Joshi' ${ }^{2 *}$ \\ ${ }^{\mathbf{1}}$ Associate Professor, ${ }^{\mathbf{2}}$ Assistant Professor, Dept. of Orthopaedics, Ashwini Rural Medical College, Hospital and Research \\ Centre, Kumbhari, Solapur, Maharashtra, India \\ *Corresponding Author: Milind M Joshi \\ Email: milindj80@gmail.com
}

\begin{abstract}
Introduction: With modern day epidemic of automobile accidents escalating fractures of distal end of femur will continue to be oft encountered by present day orthopedic surgeon. Considering the complex articular Anatomy of knee and close proximity to major structures, management of these fractures still poses challenge to operating surgeon. The present study undertaken taken to study fractures of distal end of femur in respect to mechanism, configuration, and management.

Materials and Methods: Thirty five patients of fractures of distal femur were selected for this study during the duration of two years. Duration of follow up ranged from 3 months to 17 months. All the patients were hospitalized and managed according to standard protocol, which included, preoperative evaluation, a period of non - weight bearing, mobilization of knee, discharge from hospital after suture removal and regular follow - up on outpatient basis.

Results: Male patients (74.29\%) were more than female (25.71\%) in the present study. Right side was predominant. One patient was having bilateral distal femoral fracture. Grade I fracture $(11.11 \%)$ is most common type found in the present study. According to Muller's classification, most common type found to be A-1 (40\%) followed by C-2 (28.6\%). In the present, majority of cases outcome shows Good (51.4\%) followed by Excellent (22.9\%), Fair (14.3\%) and Poor (11.4\%).

Conclusion: It is stable fixation and allows. Postoperatively patient motivation and aggressive physiotherapy with continuous passive motion if necessary is necessary to prevent knee stiffness.
\end{abstract}

Keywords: Distal femoral fractures, Muller's classification, Intramedullary Supracondylar nail, Knee stiffness.

\section{Introduction}

With advent of newer imaging techniques like spiral CT - scan, it has now become possible reconstruct the fracture fragments in 3 dimensions, and better understand fracture anatomy pre-operatively and thence plan operative procedure. Almost all the fractures of distal end of femur in adults require some sort internal fixation with very few indications for conservatism.

Distal femoral fractures are much less common than hip fractures and account for $7 \%$ of all femoral fractures ${ }^{1}$. If fractures of hip are excluded, $31 \%$ of femoral fractures involve the distal portion. Over the past 30 years, implants and techniques have improved. It is now recognized by most orthopedic surgeons that distal femoral fractures are best treated by reduction and surgical stabilization. Anatomic reduction of articular surface restoration of limb alignment and early mobilization, have been shown to be effective way of managing most distal femoral fractures. ${ }^{1}$ Despite advances in techniques and improvement in surgical implants, treatment of distal femoral fractures remains a challenge in many situations. Long term disability still occur in patients with extensive articular cartilage damage, marked bone communication, and severe soft tissue injury. ${ }^{2,3}$

Because of high incidence of infection, non-union, malunion, inadequate fixation and lack of proper instruments, implant as well as antibiotics, in earlier days, there was a great lack of enthusiasm towards operative management of this fracture. Then, along the principle of Watson Jones and John Charnley, the traditional management of displaced fracture supracondylar of femur. ${ }^{4}$

Keeping in mind above facts, the present study undertaken taken to study fractures of distal end of femur in respect to mechanism, configuration, and management. And also to study results of management of these fractures with intramedullary supracondylar nail, in respect to stability, mobilization, and union.

\section{Materials and Methods}

Thirty - five patients of fractures of distal femur were selected for this study during the duration of two years. Duration of follow up ranged from 3 months to 17 months. All the patients were hospitalized and managed according to standard protocol, which included, preoperative evaluation, a period of non weight bearing, mobilization of knee, discharge from hospital after suture removal and regular follow - up on outpatient basis.

\section{Preoperative Evaluation}

Every patient of distal femoral fractures is assessed systemically to assure full evaluation and so other injuries are not over looked. Particular attention must be paid to distal neurovascular status, as these fractures are close to popliteal artery. All the fractures were classified according to Muller's classification of distal femoral fractures.

Considerable swelling of thigh and knee develop shortly after injury. Care is taken to assure that skin is 
intact, skin is thoroughly examined for presence of impending open fracture, which is commonly associated with these fractures, with distal fragment displaced posteriorly and femoral shaft (proximal fragment) if pierced the quadriceps tents the skin anteriorly.

In case of compound fracture the grade of injury was ascertained by Gustallio and Anderson's classification of open fractures. All compound fractures were subjected to immediate debridment under local or general anaesthesia, and as per severity intravenous antibiotics and anti - tetanus serum. An above knee plaster splint in slight flexion is used as primary immobilization. Limb was elevated by pillow underneath leg or by elevating leg end of bed. While awaiting definitive treatment, repeated assessment of distal vascular status is made.

\section{Radiological - Evaluation}

Standard antero -posterior and lateral view of knee with femur taken, oblique views are also taken if fracture is intra - articular. Radiograph should include part of femur until proposed nail length to know the medullar canal width, for selection of proper implant.

Other Investigations: $\mathrm{Hb}$, total and differential counts, Blood urea, Sr creatinine and serum electrylytes. Chest $\mathrm{X}$ - ray, ECG, and physician consultation done for all patient above 40 years.

\section{Implants Considered:}

1. Intramedullary supracondylar nail of proper length and diameter.

2. Intelocking bolts $5 \mathrm{~mm}$

3. Partially threaded $6.5 \mathrm{~mm}$ cancellous screws.

\section{Pre-operative Planning}

Each fracture was classified according to Muller's classification. Each fracture is studied in all available views. All the major fragments and their displacements noted. Orientation of fracture geometry was obtained with special attention to intra - articular fracture geometry. Degrees of communition and displacement as well as outline of key fragments were noted.

This helps during surgery as it involved closed reduction by indirect reduction techniques and per cutaneous fixation. Implant size and type also selected over the radiograph. Assesment of nail length and size use of longer nail results in centralization of nail in isthmus and reduces its intramedullary deviation laterally or medially. ${ }^{5}$

Centering diaphysis prevents varus and valgus angulation. Nail should extend proximally from intercondylar notch so that at least two interlocking bolts can be placed distal and proximal to fracture line to achieve a stable osteosynthesis. Nail size should depend on size of medullary canal. Careful consideration should be given before using larger diameter nails in severely communited intra - articular fracture as they further displace fracture. It is always recommended to use larger diameter implant 35 suitable for patient without causing disruption at fracture site.

\section{Operative Procedure}

Materials Required: Plain radiolucent table, I.I. T.V. (Image Intensifier Television), General surgical tray, Intramedullary supracondylar nail, $5 \mathrm{~mm}$ interlocking bolts, $6.5 \mathrm{~mm}$ cancellous screws, Steinmann pin, Guide wire, Sleeve, I.M.S.C jig and set, Bolsters.

All the patients were given $1 \mathrm{gm}$ of $3^{\text {rd }}$ generation cephalosporin $1 \mathrm{hr}$ prior to surgery. Anaesthesist decided the choice of anaesthesia regional or general. After induction of anaesthesia, patient was taken on plane radiolucent table in supine position with bolster under thigh and affected leg hanging down from bolster with about 40 flexion of affected knee. Advantages of boalster are, keeps knee in flexion and so reduces the posteriorly tilted distal fragment. Boalster is directly posterior to posterorly tilted distal fragment reducing it.

Tourniquet applied to proximal thigh if necessary to avoid blood loss and to provide a blood less field. Painting was done with savlon, spirit and betadine from knee to proximal thigh. Draping was done from knee to proximal thigh.

\section{Surgical Procedure}

Reduction: Reduction is usually achieved close by knee flexion with bolster underneath lower thigh, with minimal traction. Patient in which reduction is not achieved by this upper tibial Steinmann pin is used for manipulation. Priority is for articular reduction, no compromise is done for articular congruity, and if it is not achieved closed pen reduction is done. Incision knee joint is entered through standard midline skin incision from inferior border of patella to tibial tuberosity. Patellar tendon is incised vertically in midline by splitting longitudinal fibres..$^{5}$

Small retractors are used to minimize trauma. Infrapatellar fat may need removal. Entry point is made in intercondylar notch; just $0.5 \mathrm{~cm}$ anterior to origin of posterior cruciate ligament. ${ }^{4}$ It is made with straight awl. Entry should be centralized.

With condyles in anatomic alignment to ensure that alignment will not devite into varus or valgus position. As the straight awl is postioned into intercondylar portal, AP and Lateral C - arm verify this. On AP view straight awl should be directed in neutral position with respect to condyles. In lateral view it should be parallel to anterior cortex, which is straighter once the awl is properly, it is advanced deep into metaphysis.

Awl is removed and the standard ball tipped guide wire advanced through entry Portal and proximally into shaft. Keep the guide wire parallel to anterior cortex of femur and lateral cortex of femur in other plane. Entry portal and medullary canal are enlarged with intramedullary reamers gradually.

Reaming is begun with $8 \mathrm{~mm}$ portal and medullary canal is enlarged upto a size $1 \mathrm{~mm}$ to $1.5 \mathrm{~mm}$ larger 
than the size of nail to be inserted. When reaming is completed, the nail and I.M.S.C. drill guide are assembled. The nail is advanced over the guide wire into the distal condyles. Traction is applied at this time with gentle pull behind the gastronemius with knee in 30 to $40 *$ of flexion.

Once the nail is in the distal condyles fragment, it is advanced with hand pressure over guide wire until the distal end of nail is countersunk 1-2 mm below articular surface. This is checked by disappearance of two ring marked on jig which indicates that the nail is inside. Same can be confirmed on $\mathrm{C}-$ arm by passing a $\mathrm{K}$ - wire from a small hole in jig which indicated the position of tip of nail. ${ }^{5}$

Next two to three distal interlocking bolts are placed by standard procedure. Two screws are placed proximally and are sufficient for good stability. In a 25 $\mathrm{cm}$ nail, it may be difficult to lock the proximal most hole with jig, as it may go posteriorly. The knee joint is copiously irrigated with pulse lavage. Patellar tendon repaired with absorbable sutures. It is not necessary to fill in all the holes in standard multihole nail. It is necessary to insert at least two interlocking bolts in the distal fragment (preferably three) and two in proximal fragment to achieve a stable fixation. Screws should not be placed in areas of communication.

Decision to use an open or percuaneous technique can usually be made pre - operatively. Those fractures, which are confined to metaphysical portion of distal femur without involvement of articular surface, can be fixed without opening the fracture site, Sometimes even when the \# line extends into intercondylar notch, it may be possible to achieve good closed reduction with manual traction on leg. ${ }^{4}$ In this position of canulated screw over guidewires, anterior and posterior to intended position of nail can be done with the use of percutaneous technique. Failure to obtain an accurate articular reduction is an indication of open of the fracture all intra- articular are anatomically reduced and fixed with screws. Screws can be placed lateral to medial or medial to lateral in anterior or posterior or both segments of condyles, allowing adequate space to place the nail centrally immedullary canal. Upon stabilization of intercondylar component of fracture, nailing for supracondylar \# is done as described above.

\section{Postoperative Care}

In stable fractures knee mobilization started on next day, patient is allowed non - weight bearing initially in unstable fractures - patient is allowed non weight bearing with brace insitu. Static quadriceps exercises started on next day I.V. antibiotics given for 48 hours. Suture removal done on $11^{\text {th }}$ post - operative day, crutch walking taught in hospital Patient was kept non - weight bearing until some evidence of callus was seen.

\section{Follow up:}

All patients were advised regular follow - up at monthly interval. Special attention is given to knee movements and patients treated with continuous passive motion (C.P.M.) if necessary. Fracture is assessed and patient is advised partial weight - bearing once some evidence o callus is seen.

\section{Clinical Operative Photography with X-ray}

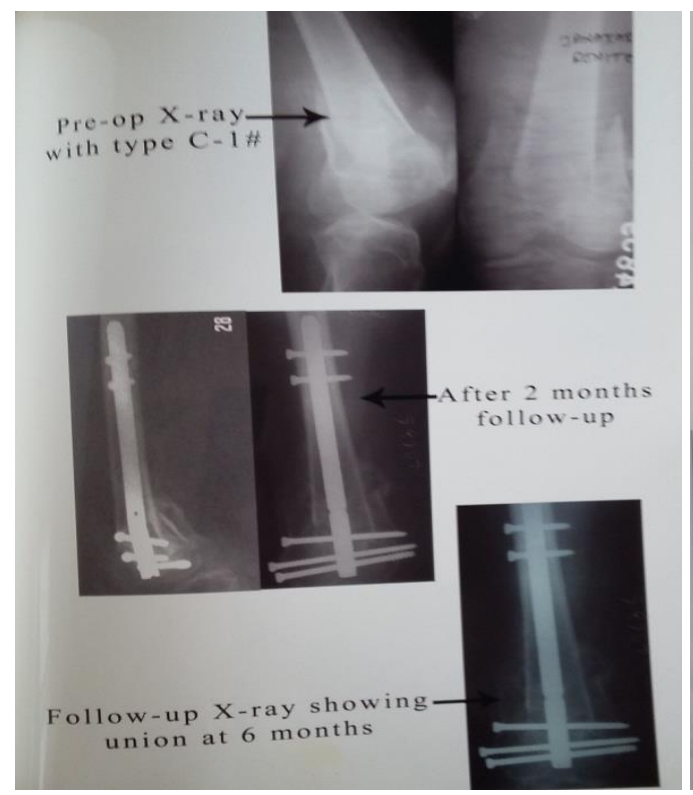

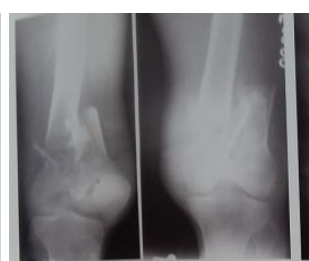

Pre-op

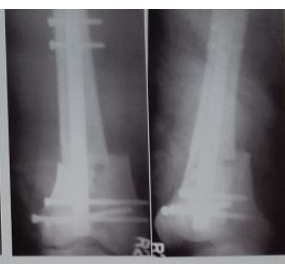

Immediate post-op

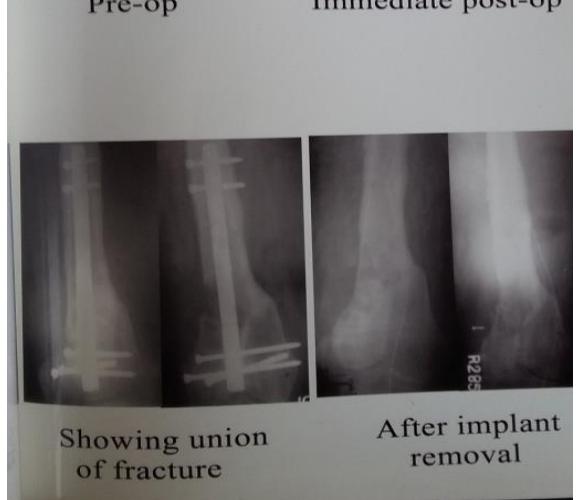

Fig. 1A: Showing Pre-operative X-ray, follow-up X-ray after 2 months and Follow-up X-ray showing union at 6 months; B: Showing Pre-operative X-ray, Immediate post operative X-ray, Union of fracture, and after removal of implant 

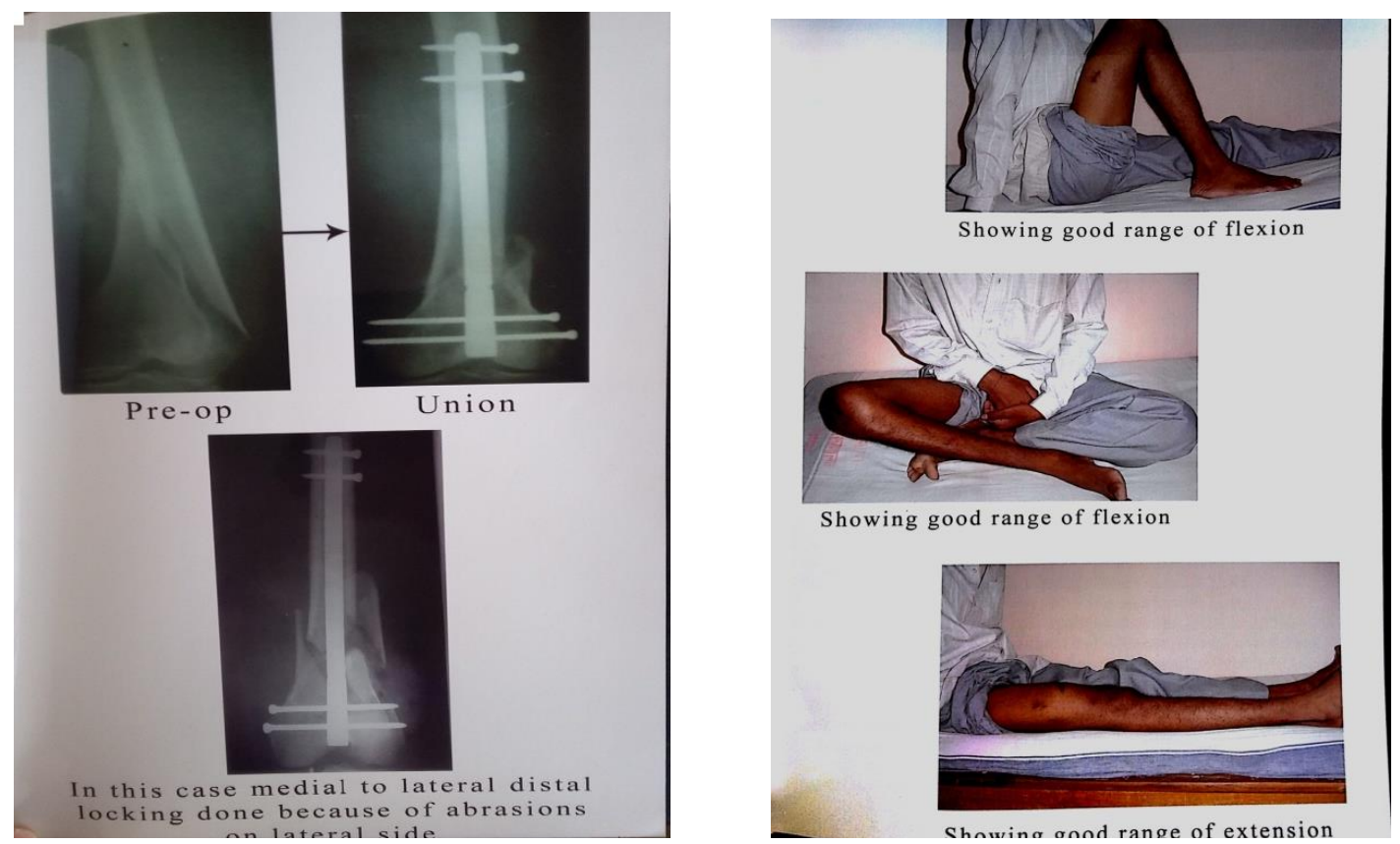

Fig. 2A: Showing Pre-operative X-ray, Union and medial to lateral distal locking done because of abrasions on lateral side; B: Patient showing good range of flexion and extension

\section{Criteria for Assessment of Results}

Evaluation scale developed by Sanders et. ${ }^{5} \mathrm{Al}$ chosen because it emphasis the most important patient outcome factors pain and knee range of movements

\section{Sanders Clinical Rating: ${ }^{5}$}

Maximum points -40

\begin{tabular}{|l|c|}
\hline \multicolumn{1}{|c|}{ Result } & Points \\
\hline Excellent & $36-40$ \\
\hline Good & $25-35$ \\
\hline Fair & $16-25$ \\
\hline Poor & $0-15$ \\
\hline
\end{tabular}

\section{Statistical Analysis}

Descriptive statistics such as mean, SD and percentage was used. Microsoft excel was used to generate tables and graphs.

\section{Results}

Age Distribution: Majority of patients were belongs to age group 31-40yrs $(37.14 \%)$ followed by $21-30 \mathrm{yrs}$ (28.57\%), 41-50yrs (20\%), 51-60yrs (8.57\%) and 61$70 \mathrm{yrs}(5.71 \%)$. Mean age group was 36.45 years

Sex Distribution: Male patients (74.29\%) were more than female $(25.71 \%)$ in the present study.

Table 1: Shows side of fracture

\begin{tabular}{|l|c|c|}
\hline \multicolumn{1}{|c|}{ Side } & No. of cases & Percentage (\%) \\
\hline Right & 19 & 54.3 \\
\hline Left & 16 & 45.7 \\
\hline Total & 36 & 100 \\
\hline
\end{tabular}

Right side was predominant. One patient was having bilateral distal femoral fracture.
Table 2: Type of injury

\begin{tabular}{|l|c|c|}
\hline \multicolumn{1}{|c|}{ Type } & No. of cases & Percentage (\%) \\
\hline Closed & 29 & 80.56 \\
\hline Open & 7 & 19.44 \\
\hline Total & 26 & 100 \\
\hline
\end{tabular}

Majority of the injury is closed (80.56\%).

Table 3: Grading of compound fracture [Gustallio Anderson's]

\begin{tabular}{|l|c|c|}
\hline \multicolumn{1}{|c|}{ Type } & No. of cases & Percentage (\%) \\
\hline Grade I & 4 & 11.11 \\
\hline Grade II & 3 & 8.33 \\
\hline Grade III & 0 & 00.00 \\
\hline Total & 7 & 19.44 \\
\hline
\end{tabular}

Grade I fracture (11.11\%) is most common type found in the present study.

Table 4: Type of fracture according to Muller's classification

\begin{tabular}{|l|c|c|c|}
\hline S. No. & $\begin{array}{c}\text { Fracture } \\
\text { type }\end{array}$ & $\begin{array}{c}\text { No. of } \\
\text { cases }\end{array}$ & $\begin{array}{c}\text { Percentage } \\
(\mathbf{\%})\end{array}$ \\
\hline 1 & $\mathrm{~A}-1$ & 14 & 40 \\
\hline 2 & $\mathrm{~A}-2$ & 4 & 8.6 \\
\hline 3 & $\mathrm{~A}-3$ & 2 & 5.7 \\
\hline 4 & $\mathrm{~B}-1$ & 0 & 00.00 \\
\hline 5 & $\mathrm{~B}-2$ & 0 & 00.00 \\
\hline 6 & $\mathrm{~B}-3$ & 0 & 00.00 \\
\hline 7 & $\mathrm{C}-1$ & 6 & 17.1 \\
\hline 8 & $\mathrm{C}-2$ & 10 & 28.6 \\
\hline 9 & $\mathrm{C}-3$ & 0 & 00.00 \\
\hline Total & & 35 & 100 \\
\hline
\end{tabular}

According to Muller's classification, most common type found to be A-1 (40\%) followed by C-2 (28.6\%) 
Table 5: Associated injuries

\begin{tabular}{|l|c|c|c|}
\hline S. No. & $\begin{array}{c}\text { Associated } \\
\text { fractures }\end{array}$ & No & $\begin{array}{c}\text { Percentage } \\
\text { (\%) }\end{array}$ \\
\hline 1 & Distal Radius & 1 & 2.85 \\
\hline 2 & Tibial Plateau & 1 & 2.85 \\
\hline 3 & Humerus & 1 & 2.85 \\
\hline 4 & Shaft Tibia & 1 & 2.85 \\
\hline 5 & Shaft Femur (opp) & 1 & 2.85 \\
\hline \multicolumn{2}{|l}{ Total } & 5 & $14.29 \%$ \\
\hline
\end{tabular}

Out of 35 patients 5 cases (14.29\%) have associated other fractures.

Table 6: Outcome of study

\begin{tabular}{|l|c|c|}
\hline \multicolumn{1}{|c|}{ Grade } & No. of patients & Percentage \\
\hline Excellent & 8 & 22.9 \\
\hline Good & 19 & 51.4 \\
\hline Fair & 5 & 14.3 \\
\hline Poor & 4 & 11.4 \\
\hline
\end{tabular}

In the present, majority of cases outcome shows Good (51.4\%) followed by Excellent (22.9\%), Fair (14.3\%) and Poor $(11.4 \%)$

\section{Discussion}

Distal femoral fractures are uncommon injuries much less common than hip fractures and account for about $7 \%$ of all femoral fractures. ${ }^{1}$

Gellmann and green ${ }^{7}$ has treated 24 patients with distal femoral fractures with intramedullary supracondylar nail and they have excellent results in $16.67 \%$ and good results in $62.50 \%$.

Also A. Saw and C.P. Law $^{8}$ reported their experience with 13 patients and their results were excellent in $46.15 \%$ and good in $23 \%$ patients.

The present study of management of supracondylar fractures by intramedullary supracondylar nail (I.M.S.C.) has been made with aim to try and reduce morbidity. This series has 36 distal femoral fractures treated in 35 patients with intramedullary supracondylar nail.

Male predominance was seen as mechanism of injury high - energy trauma in most of the patients and since males is more susceptible due to their activity. 26 patients or $74.28 \%$ were male and 9 patients or $25.72 \%$ were female, osteoporosis being commonly found in elderly female with distal femoral fractures.

In present study, peak incidence was observed in age of 31-40 years i.e. 13 of 35 patients (37.14\%), this can be explained as in this age group, activity of livelihood, job opportunities and vehicular mobility predisposed them to high energy vehicular accidents. Average age in our study was 36.45 years, youngest patient was of 23 years and oldest patient being of 70 years. Average age in series of Gellmann and Green ${ }^{7}$ was 50 years.

In study of Lucas and Henry ${ }^{9}$ average age was 39 years with range from 16 to 69 years right - sided fractures were marginally more than left side (19 out of
36) $80.555 \%$ of fractures in our series had closed fractures means 29 out of 36 fractures were closed.

In series of Gellmann and Green ${ }^{7} 18$ out of 26 fractures were closed $(69.23 \%)$, in series of Lucas Seligson and Henry ${ }^{9} 15$ of 24 patients were of closed fractures $(62.50 \%) .19 .45 \%$ of distal femoral fractures in our series were compound and 4 were of grade I, and 3 of grade II. None of grade III fractures treated with supracondylar nailing in our series.

Mode of injury was high - energy trauma in most patients, of which 16 patients have comminuted \#s. $79 \%$ of patients of Lucas Seligson and Henry's series ${ }^{9}$ were of high - energy trauma. Also 50\% of patients of Gellmann and Green's series 08 were having high energy trauma [13 of 26\#s] in our series all the fractures were classified according to Muller's classification. Accordingly extra - articular [type A] fractures are seen in 20 patients [55.66\%] and intra-articular type - C fractures seen in 16 patients [ $44.44 \%$ ].

Of type A \#s, 14 cases were of type A-1 which consists of predominant group of our series, 4 patients were of type A-2 and only two patients were having comminuted extra- articular \#s [A-3]. Of the 16 intra articular \#s type C-2 dominated with 10 cases indicating high energy of trauma, 6 patients were having ' $\mathrm{T}$ ' - ' $\mathrm{Y}$ ' type of intra - articular \#s [type $\mathrm{C}$ ] and none of the patients with intra- articular communication was treated with supracondylar nail.

Only three [8.33\%] of patients of our series were treated with open reduction to get an anatomical intra articular reduction, no compromise was done for getting anatomical articular congruity. Average time for definitive stabilization of \#s in our series was 3 days, ranging from 1 day to 8 days after injury. In cases of intra - articular [type C] fractures, basic strategy was to assemble condylar block and then fix it to femoral shaft with nail.

In present study, we have achieved closed reduction I 13 of 16 intra- articular fractures. Only three patients needed open reduction for it. Canulated cancellous screws and $\mathrm{K}$ - wires are used to fix condylar block before taking entry through intercondylar notch.

$14.22 \%$ of patients i.e. 5 of 35 were having associated injuries. One each of distal radius \#, tibial plateau \#, \# shaft humerus, \# shaft tibia, and one patient had contra lateral \# shaft femur. In our series of 36 cases 33 patients were operated through minimal invasive technique by midline patellar tendon splitting incision. Splitting of patellar tendon for insertion of nail did not appear to cause any symptomatic or functional problems with knee.

Nails used were of variable lengths varying from $20 \mathrm{~cm}$ to $35 \mathrm{~cm}, 6.5 \mathrm{~mm}$ cancellous screws are used for fixation of condylar block in case of type $\mathrm{C}$ fractures. 5 $\mathrm{mm}$ interlocking bolts were used for interlocking with minimal two distal locking bolts. In general, postoperative management consisted of the placement 
of knee immobilizer immediately after surgery. Institution of continuous passive motion as well as isometric and range of movement exercises started as early as possible, within the its postoperative week. Success in this physical therapy varied with patient's reliability.

Patients were advised touchdown weight bearing until there was radiological sign of callous formation. Partial weight bearing was usually begun as $8 \mathrm{wks}$ and full weight bearing was begun by 16 wising Gellmann ${ }^{7}$ et al series partial weight - bearing was begun at $8 \mathrm{wks}$ and full weight - bearing at 12 wks.

Fracture healing was determined by presence of bridging callus on anterio - posterior and lateral radipgraphs and absence of tenderness or motion at \# site. Average union time in our series was 16 wks. In Gellmann ${ }^{7}$ et al series also average time for union was 16 wks. In Saw et al series 14.4 months ws the average time for union. In our series out of 36 cases union seen in 32 cases two cases developed delayed union, which were later treated with bone grafting.

Load sharing mechanism of intramedullary fixation promotes secondary bone healing \& morcelised bone from medullary reaming extravasated into \# site serves as bone graft. ${ }^{8}$ All these fractures likely contributed to good union rate and low incidence of soft tissue infection. ${ }^{8}$

Angular malunion may develop due to inadequate reduction or subsequent displacement during bone healing. Moment arm of varus - valgus bending force is significantly reduced with intramedullary nailing. More over intramedullary position of nail also provides 3 point fixation of \#, to prevent flexion extension displacement of distal fragment. ${ }^{8}$

\section{Conclusion}

From the present study, it can be conclude that, Supracondylar nailing is safe and optional tool for fixation of many supracondylar and intercondylar fractures. With minimal disruption of soft tissues and good purchase of the distal fragment, this approach provides stable fracture, allowing early joint mobilization. In case of intraarticular fractures joint congruity has to be achieved by all possible means, if necessary by open reduction. Rate of union is high with a low incidence of complications. Simplicity of procedure also facilitates fracture fixation in patients with multiple trauma including those with multiple fractures. Malunion is rare as moment arm of varus / valgus bending force is significantly reduced with intramedullary nailing. Load sharing mechanism of intramedullary nailing, promotes secondary bone healing. It is stable fixation and allows. Postoperatively patient motivation and aggressive physiotherapy with continuous passive motion if necessary is necessary to prevent knee stiffness.
It requires several studies and longer follow - up to better evaluate technique and to know long term results on function of knee joint.

\section{Conflict of Interest: Nil}

\section{References}

1. Wiss DA: Supracondylar and intercondylar fractures of femur. In Rockwood CA, Green DP, Bucholz RW et al eds. Rockwood and Green fractures in adults. 1991(1):1972-1999.

2. Sanders R, Kovel KJ, Dipas Quale T, Helfet N. Retrograde reamed femoral nailing. J. Orthop Trauma. 1993;7(4):293-302.

3. Schatzakar: Fractures of distal femur revisited. Clinic orthop. 1998;347:43-56.

4. John C. The closed treatment of common fractures. 3 ed; 2003:197-204.

5. Tanna DD: Interlocking nailing $2^{\text {nd }}$ edi: 2004:123-134.

6. Sanders and Swintkowski. Double-plating of comminuted, unstable fractures of the distal part of the femur. JBJS. 1993;73(3):341-346.

7. Gellmann, Paiement, Green: treatment of supracondylar femoral fractures with retrograde intramedullary nail: CORR. 1996; 332:90-97.

8. A Saw, CP Lau. Supracondylar nailing for difficult distal femur fractures. Journal of Orthopaedic Surgery. 2003;11 (2):141-147.

9. Lucas - Seligson and Henry: Intramedullary supracondylar nailing of femoral fractures. CORR. 1993;296:200-206.

How to cite this article: Yemul S, Joshi M. M. A study of management of distal femoral fractures with intramedullary supracondylar nail. Indian $\mathbf{J}$ Orthop Surg. 2018;4(4):362-367. 